\title{
Throughput Fairness for Wireless Powered Cognitive Hybrid Active-Passive Communications
}

\author{
Shuang Fu $\mathbb{D}$, Chenyang Ding $\mathbb{D}$, and Peng Jiang $\mathbb{E}$ \\ Institute of Information and Electrical Engineering, Heilongjiang Bayi Agricultural University, Daqing, China \\ Correspondence should be addressed to Shuang Fu; fushuang_dq@163.com
}

Received 24 November 2021; Revised 2 January 2022; Accepted 17 January 2022; Published 9 February 2022

Academic Editor: Liqin Shi

Copyright (c) 2022 Shuang Fu et al. This is an open access article distributed under the Creative Commons Attribution License, which permits unrestricted use, distribution, and reproduction in any medium, provided the original work is properly cited.

\begin{abstract}
In this paper, we consider a backscatter communication (BackCom)-based cognitive network that consists of one primary transmitter, one primary receiver, multiple secondary transmitters (STs), and one secondary receiver (SR). Each ST operates in the BackCom or energy harvesting model. Our goal is to jointly optimize the energy harvesting and backscatter time, the transmit power of the primary transmitter, and the power reflection coefficient of each ST to maximize the sum throughput of all the STs under a nonlinear energy harvesting model while satisfying multiple constraints, i.e., the energy causality of each ST, the quality of service of the primary transmitter, etc. The formulated problem is nonconvex due to the coupled variables and is hard to solve. In order to address this problem, we decouple partially coupled variables by using the properties of the objective function and constructing auxiliary variables, and the remaining coupled variables are decoupled via successive convex approximation (SCA). On this basis, a SCA-based iterative algorithm is developed to solve the formulated problem. Simulation results are provided to support our work.
\end{abstract}

\section{Introduction}

Increasing demands for intelligent services have boosted the attention of Internet of Things (IoT), where massive tiny IoT devices should be deployed for connecting the physical environment and cyberspace seamlessly [1]. This poses an urgent need for developing a high spectrum efficient communication technology in the era of IoT networks. In this context, cognitive radio has been proposed, where the IoT nodes are allowed to share the spectrum with the primary users $[2,3]$. Despite the improvement of spectrum efficiency for tiny IoT nodes, most of them still suffer from the short life span as they are powered by a limited battery capacity [4]. In order to address this challenge, wireless powered hybrid active-passive communication $[5,6]$ has been integrated into the cognitive radio, yielding a wireless powered cognitive hybrid active-passive communication network $[7,8]$.

Until now, there are considerable works on the design of resource allocation schemes for wireless powered cognitive hybrid active-passive communication network. In [9], the authors proposed to maximize the rate of an IoT node by jointly optimizing the energy harvesting time, the backscattering time, and the active communication time, while satisfying that the consumed energy of an IoT node does not exceed the harvested energy. Subsequently, this work was extended into a scenario with multiple IoT nodes [10], where the main focus was to find the optimal tradeoff among the energy harvesting time, the backscattering time, and each IoT node's active communication time. In the above two works $[9,10]$, the rate of the considered backscatter communication was assumed to be a constant. In [11], the authors considered another wireless powered cognitive hybrid active-passive communication network, where two different backscatter communications are introduced, and proposed an optimal time allocation scheme to maximize the throughput of the IoT node. Considering that energy efficiency is an important performance metric in wireless communications, the authors in [12] maximized the energy efficiency of an IoT node while considering the primary interference and imperfect spectrum sensing constraints. In [13], the authors considered multiple IoT nodes and 
proposed to maximize the energy efficiency of all the IoT nodes by jointly optimizing the time and power resources, subject to the minimum throughput requirement of each IoT node and the energy causality constraint of each IoT node.

Although the above works have provided a solid foundation for understanding the resource allocation in wireless powered cognitive hybrid active-passive communication networks, some gaps still exist. First, the above works mainly ignored the interference introduced by the IoT node. More specifically, for the backscattering time, the interference from the IoT node to the primary was ignored. Second, fairness among IoT nodes has not been considered. Third, in the practical energy harvester, the output power is a nonlinear function with respect to the input power [14], while this nonlinearity has been ignored in most of the existing works. Motivated by the above observations, in this paper, we study the throughput fairness in a wireless powered cognitive hybrid active-passive communication network that consists of multiple backscatter devices (BDs) and backscatter receivers (BRs), one primary transmitter $(\mathrm{PT})$, and one primary receiver (PR). The main contributions are summarized as follows:

(i) A throughput fairness problem is formulated. In particular, this problem maximizes the minimum throughput that achieved all IoT nodes by jointly optimizing the transmit power and time of the primary transmitter (PT), the BDs' time sharing among energy harvesting (EH), backscatter communication (BackCom) and active communications, and the power reflection coefficient and transmit power of each BD subject to the quality of service (QoS), energy causality, and transmit power constraints.

(ii) We develop an iterative algorithm to solve the formulated problem. More specifically, we first derive the optimal transmit power of the PT in a closed form via contradiction and then construct a series of auxiliary variables to decouple the coupled variables. Lastly, successive convex approximation (SCA) is leveraged to address the nonconvex QoS constraint. On this basis, an efficient iterative algorithm is proposed to solve the formulated problem.

(iii) We provide computer simulation to verify the quick convergence of the proposed algorithm and demonstrate that the fairness throughput can be ensured by our proposed scheme.

\section{System Model}

2.1. Basic Settings of the Considered Network. In this paper, the wireless powered cognitive hybrid active-passive communication and the wireless powered cognitive network with hybrid active-passive communications are used interchangeably, which is shown in Figure 1. Specifically, the whole network consists of one PT, one PR, $K$ BDs, and their receivers, where the $\mathrm{PT}$ broadcasts its signals to the $\mathrm{PR}$ for

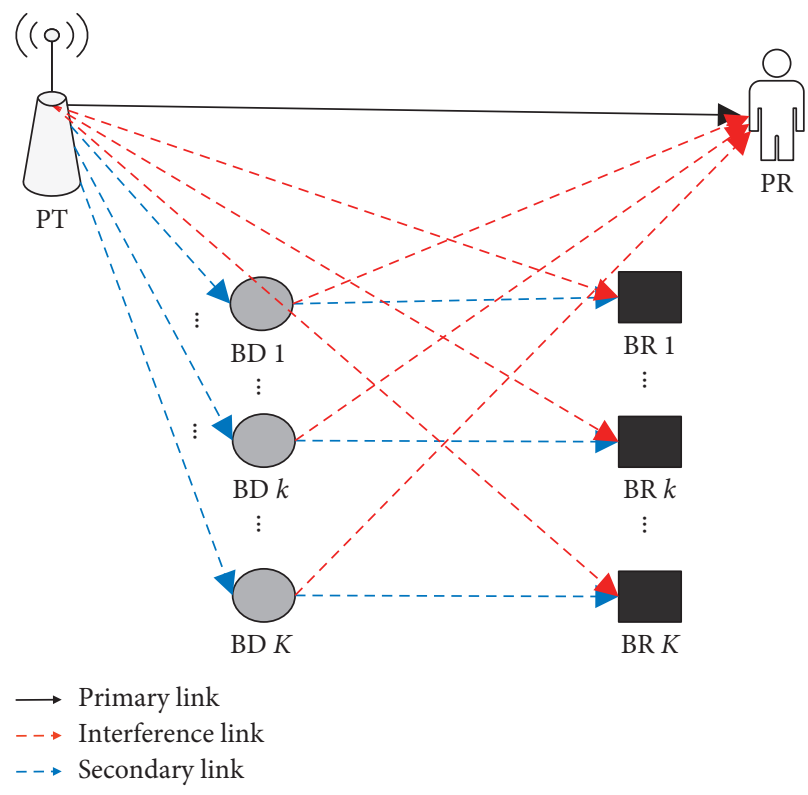

Figure 1: BackCom-based cognitive networks and its frame structure.

the primary transmission, and the PT's signals are also exploited by the $K$ BDs for energy harvesting $(\mathrm{EH})$ and BackCom. We note that when the PT is idle, each BD can also use its harvested energy to transmit its information by active communications. That is to say, each BD can backscatter its received signals for passive communications when PT is busy and use its harvested energy for active communications when PT is idle. We note that each BD only harvests energy from the energy signals from the PT since the energy harvested from other nodes during active and backscatter communications is very weak. All the devices are assumed to be equipped with a single antenna and always work in the half-duplex mode. The reasons are as follows: we note that both EH and BackCom are particularly applicable to wireless sensor networks for each sensor node's information transmission, where it may be difficult for low-cost small wireless sensor nodes to have multiple antennas and work at the full-duplex mode. Meanwhile, all devices with a single antenna have also been assumed in many related recent works. All the $\mathrm{BDs}$ are energy constrained, where each $\mathrm{BD}$ uses its harvested energy in each transmission block to support its energy consumption so that the operation time of each $\mathrm{BD}$ is prolonged. We assume that all channels are quasistatic fading. Let $h_{p}$ and $g_{k}(k \in \mathscr{K}=\{1,2, \ldots, K\})$ denote the channel gains from the PT to the PR and the $k$-th $\mathrm{BD}$, respectively. We denote the channel gains from the $k$-th $\mathrm{BD}$ to its receiver and the $\mathrm{PR}$ as $f_{k}$ and $f_{p, k}$. The channel gain from the $\mathrm{PT}$ to the $k$-th $\mathrm{BD}$ 's receiver (BR) is expressed as $h_{k}$. In the beginning of each transmission block, the channel estimation is adopted by the PT so as to perfectly know the channel state information (CSI) of all links, and then the PT can determine the optimal resource allocation scheme according to the achieved CSI, and the optimal resource allocation scheme can be performed successively. In this work, we clarify how to obtain all the channel gains of 
all links as follows: the channel gain from the PT to the PR (or the $k$-th BR) can be obtained by performing the existing advanced channel estimation methods, e.g., least-square estimation, etc. The value of the product of the forward channel gain and the backward channel gain from the $k$-th $\mathrm{BD}$ to the $\mathrm{PT}$ (or the $k$-th $\mathrm{BR}$, the PR) can be obtained by performing least-square estimation. Due to the channel reciprocity, the forward channel gain equals the backward channel gain, and hence, the channel gain from the $k$-th $\mathrm{BD}$ to the PT (or the $k$-th $\mathrm{BR}$, the PR) can be obtained.

2.2. Frame Structure. As shown in Figure 2 , let $T$ denote the duration of each transmission block. For the primary transmission, the whole block can be divided into two phases. In the first phase, the PT performs information transmission. Let $\beta$ with $0 \leq \beta \leq T$ denote the transmission time of the primary transmission. In this phase, $K$ BDs first work in the EH mode, where all the received signals are used to harvest energy and then take turns to work in the BackCom mode. In particular, let $t_{e}$ be the $\mathrm{EH}$ time for all $\mathrm{BDs}$ and $t_{k}$ be the backscattering time for the $k$-th BD. Then, we have $t_{e}+\sum_{k=1}^{K} t_{k} \leq \beta$. We note that in the subphase $t_{k}$, the $k$-th BD performs BackCom, while the other BDs still work in the $\mathrm{EH}$ mode in order to improve its harvested energy. In the second phase with duration $T-\beta$, the PT stops its information transmission, and all the BDs can use their harvested energy to transmit their information. In order to avoid co-channel interference among BDs, all the BDs take turns to perform information transmission. Let $\tau_{k}$ denote the transmit time of the $k$-th $\mathrm{BD}$ in this phase. Then, we have $\sum_{k=1}^{K} \tau_{k} \leq T-\beta$.

In the following part, we will clarify how the system works from both the primary transmission and BDs' transmissions. In the subphase $t_{e}$, we denote $P_{0}$ as the transmit power of the PT. Then, the received signals at the $k$-th $(k \in \mathscr{K}=\{1,2, \ldots, K\})$ BD can be given by

$$
y_{\mathrm{BD}}^{k}=\sqrt{P_{0} g_{k}} x_{p}+N_{\mathrm{BD}},
$$

where $x_{p}$ with $\mathbb{E}\left[\left|x_{p}\right|^{2}\right]=1$ expresses the information transmitted by the PT to the PR, and $N_{\mathrm{BD}}$ is the additive white Gaussian noise (AWGN) at the $k$-th $\mathrm{BD}$ with mean zero and variance $\sigma^{2}$.

In this work, a nonlinear EH model proposed in [14] is considered to characterize the nonlinearity of practical $\mathrm{EH}$ circuits accurately. The reason of considering this nonlinear EH model is as follows: firstly, according to [14], the nonlinear EH model proposed in [14] is very accurate, even more accurate than the existing nonlinear EH models. Secondly, the use of the nonlinear EH model proposed in [14] removes the difficulty caused by the nonlinear $\mathrm{EH}$ model since we can prove that the nonlinear EH model proposed in [14] is concave by using the properties of practical EH circuits, which greatly reduces the difficulty of solving the formulated optimization problem. Then, the harvested energy at the $k$-th $\mathrm{BD}$ in this subphase can be calculated as
Secondary Transmission $T$

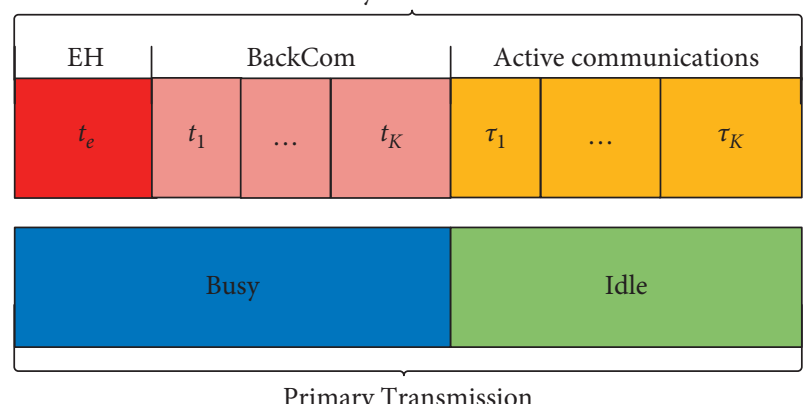

FIGURE 2: Frame structure for the considered network.

$$
E_{e}^{k}=t_{e} \phi_{k}\left[P_{0} g_{k}\right],
$$

where $\phi_{k}[x]=\left(\left(a_{k} x+d_{k}\right) /\left(x+v_{k}\right)\right)-\left(d_{k} / v_{k}\right), a_{k}, d_{k}$, and $v_{k}$ are the given parameters of the considered nonlinear EH model at the $k$-th BD and may be different for different BDs.

For the primary transmission, the received signals at the PR can be expressed as

$$
y_{\mathrm{PR}}^{e}=\sqrt{P_{0} h_{p}} x_{p}+N_{\mathrm{PR}},
$$

where $N_{\mathrm{PR}}$ is the AWGN at the PR with mean zero and variance $\sigma^{2}$. Correspondingly, the achievable throughput at the PR in this subphase can be calculated as

$$
C_{e}^{p}=t_{e} W \log _{2}\left(1+\left(\frac{P_{0} h_{p}}{\sigma^{2}}\right)\right),
$$

where $W$ is the bandwidth of the whole system.

In the subphase $t_{k}$, let $\alpha_{k}$ with $0 \leq \alpha_{k} \leq 1$ denote the power reflection coefficient of the $k$-th $\mathrm{BD}$, which decides how many signals are received at the $k$-th $\mathrm{BD}$ to be backscattered. We note that the rest signals will be flowed into the $\mathrm{EH}$ circuit of the $k$-th $\mathrm{BD}$ for $\mathrm{EH}$. Accordingly, the received signals at the $\mathrm{BR}$ in the subphase $t_{k}$ can be represented as

$$
y_{\mathrm{BR}}^{k}=\sqrt{\alpha_{k} P_{0} g_{k} f_{k}} x_{p} x_{b, k}+\sqrt{P_{0} h_{k}} x_{p}+N_{\mathrm{BR}},
$$

where $x_{b, k}$ with $\mathbb{E}\left[\left|x_{b, k}\right|^{2}\right]=1$ is the information transmitted by the $k$-th $\mathrm{BD}$, and $N_{\mathrm{BR}}$ is the AWGN at the BR with mean zero and variance $\sigma^{2}$.

It can be observed from (5) that each BD's transmission suffers from the co-channel interference to the primary transmission, leading to a reduction in the achievable throughput of the $k$-th $\mathrm{BD}$ via BackCom. Besides, owing to the double path loss fading of the BD's transmission, the signal power from the PT is always higher than that from the $\mathrm{BD}$. In order to decode $x_{b, k}$ successfully, the successive interference cancellation (SIC) technology is performed at the $k$-th BR. Specifically, the BR should decode the PT's transmitted information $x_{p}$ first by treating $\sqrt{\alpha_{k} P_{0} g_{k} f_{k}} x_{p} x_{b, k}$ as interference and then cancel the interference $\sqrt{P_{0} h_{k}} x_{p}$ since both $h_{k}$ and $x_{p}$ are known by the BR. On this basis, the transmitted information of the $k$-th BD $x_{b, k}$ can be decoded.

Based on (5), we can express the signal to interference plus noise ratio (SINR) at the BR for decoding $x_{p}$ as 


$$
\gamma_{b, k}^{p}=\frac{P_{0} h_{k}}{\alpha_{k} P_{0} g_{k} f_{k}+\sigma^{2}} .
$$

In order to ensure that the BR can decode $x_{p}$ successfully, the following inequality should hold, given by

$$
\gamma_{b, k}^{p} \geq \gamma_{t h}
$$

where $\gamma_{\text {th }}$ is the given threshold for decoding $x_{p}$, indicating the minimum SINR requirement for decoding $x_{p}$.

When $\gamma_{b, k}^{p} \geq \gamma_{\text {th }}$ holds, we can perform the SIC technology, and then the signal to noise ratio (SNR) at the BR for decoding $x_{b, k}$ is given by

$$
\gamma_{b, k}=\frac{\alpha_{k} P_{0} g_{k} f_{k}}{\epsilon P_{0} h_{k}+\sigma^{2}},
$$

where $\epsilon$ with $0 \leq \epsilon \leq 1$ denotes the interference cancellation factor.

Correspondingly, the achievable throughput at the $k$-th $\mathrm{BR}$ in this subphase can be calculated as

$$
\begin{aligned}
C_{b, k} & =t_{k} W \log _{2}\left(1+\xi \gamma_{b, k}\right) \\
& =t_{k} W \log _{2}\left(1+\frac{\xi \alpha_{k} P_{0} g_{k} f_{k}}{\epsilon P_{0} h_{k}+\sigma^{2}}\right),
\end{aligned}
$$

where $\xi$ denotes the performance gap reflecting the real modulation $[5,6]$.

For the $k$-th $\mathrm{BD}$, its harvested energy is given by

$$
E_{k}^{b}=t_{k} \phi_{k}\left[P_{0} g_{k}\left(1-\alpha_{k}\right)\right]
$$

Then, in the end of the first phase, the total harvested energy at the $k$-th $\mathrm{BD}$ can be computed as

$$
\begin{aligned}
E_{k}= & \left(\beta-t_{k}\right) \phi_{k}\left[P_{0} g_{k}\right]+t_{k} \phi_{k}\left[P_{0} g_{k}\left(1-\alpha_{k}\right)\right] \\
= & \left(\beta-t_{k}\right)\left(\frac{a_{k} P_{0} g_{k}+d_{k}}{P_{0} g_{k}+v_{k}}-\frac{d_{k}}{v_{k}}\right) \\
& +t_{k}\left(\frac{a_{k} P_{0} g_{k}\left(1-\alpha_{k}\right)+d_{k}}{P_{0} g_{k}\left(1-\alpha_{k}\right)+v_{k}}-\frac{d_{k}}{v_{k}}\right) .
\end{aligned}
$$

As for the primary transmission, it also suffers from the co-channel interference from the $k$-th $\mathrm{BD}$, and the received signals at the $\mathrm{PR}$ in the subphase $t_{k}$ are expressed as

$$
y_{\mathrm{PR}}^{k}=\sqrt{P_{0} h_{p}} x_{p}+\sqrt{\alpha_{k} P_{0} g_{k} f_{p, k}} x_{p} x_{b, k}+N_{\mathrm{PR}} .
$$

Since the signal power of the PT is higher than that of the $k$-th $\mathrm{BD}$, the $\mathrm{PR}$ will decode $x_{p}$ first by treating $\sqrt{\alpha_{k} P_{0} g_{k} f_{p, k}} x_{p} x_{b, k}$ as interference. Accordingly, the SINR at the PR for decoding $x_{p}$ is given by

$$
\gamma_{k}^{p}=\frac{P_{0} h_{p}}{\alpha_{k} P_{0} g_{k} f_{p, k}+\sigma^{2}} .
$$

Then, the achievable throughput at the PR in the subphase $t_{k}$ is determined by

$$
\begin{aligned}
C_{k}^{p} & =t_{k} W \log _{2}\left(1+\gamma_{k}^{p}\right) \\
& =t_{k} W \log _{2}\left(1+\frac{P_{0} h_{p}}{\alpha_{k} P_{0} g_{k} f_{p, k}+\sigma^{2}}\right) .
\end{aligned}
$$

When the PT is idle, each BD uses its harvested energy to transmit information. Let $p_{k}$ denote the transmit power of the $k$-th $\mathrm{BD}$ in the subphase $\tau_{k}$. Then, the achievable throughput of the $k$-th $\mathrm{BD}$ in the subphase $\tau_{k}$ is given by

$$
C_{a, k}=\tau_{k} W \log _{2}\left(1+\frac{p_{k} f_{k}}{\sigma^{2}}\right)
$$

\section{Throughput Fairness for Wireless Powered Cognitive Hybrid Active-Passive Communications}

In this section, we study the throughput fairness among different BDs for wireless powered cognitive networks with hybrid active-passive communications by designing an optimal resource allocation scheme. In particular, we formulate a throughput fairness optimization problem by jointly optimizing the transmit power and time of the PT, the $\mathrm{EH}$ time, the BackCom time, and power reflection coefficients of BDs, and the transmit power and time of each $\mathrm{BD}$, subject to multiple constraints, i.e., QoS, energy causality, transmit power, and power reflection coefficient constraints. Then, an efficient iterative algorithm is developed to solve it.

\subsection{Problem Formulation}

3.1.1. Optimization Objective. The optimization objective is to guarantee the throughput fairness among different BDs. Toward this end, a max-min approach is adopted $[6,15]$. Thus, we determine the optimization objective as maximizing the minimum achievable throughput of each BD. For the $k$-th $\mathrm{BD}$, its total achievable throughput in the whole transmission block can be computed as

$$
\begin{aligned}
C_{\mathrm{tot}}^{k} & =C_{b, k}+C_{a, k} \\
& =t_{k} W \log _{2}\left(1+\frac{\xi \alpha_{k} P_{0} g_{k} f_{k}}{\epsilon P_{0} h_{k}+\sigma^{2}}\right)+\tau_{k} W \log _{2}\left(1+\frac{p_{k} f_{k}}{\sigma^{2}}\right) .
\end{aligned}
$$

On this basis, the optimization objective is determined by $\min _{k} C_{\text {tot }}^{k}$.

3.1.2. QoS Constraint for the Primary Transmission. This constraint is to ensure that the achievable throughput of the $\mathrm{PT}$ is not less than its minimum required throughput. Based on (4) and (14), the achievable throughput of the PT can be computed as 


$$
\begin{aligned}
C_{p}= & C_{e}^{p}+\sum_{k=1}^{K} C_{k}^{p} \\
= & t_{e} W \log _{2}\left(1+\frac{P_{0} h_{p}}{\sigma^{2}}\right) \\
& +\sum_{k=1}^{K} t_{k} W \log _{2}\left(1+\frac{P_{0} h_{p}}{\alpha_{k} P_{0} g_{k} f_{p, k}+\sigma^{2}}\right) .
\end{aligned}
$$

Let $C_{\text {min }}$ denote the minimum required throughput of the primary transmission. Then, the QoS constraint for the primary transmission can be expressed as

$$
\begin{aligned}
C_{p}= & t_{e} W \log _{2}\left(1+\frac{P_{0} h_{p}}{\sigma^{2}}\right) \\
& +\sum_{k=1}^{K} t_{k} W \log _{2}\left(1+\frac{P_{0} h_{p}}{\alpha_{k} P_{0} g_{k} f_{p, k}+\sigma^{2}}\right) \geq C_{\min } .
\end{aligned}
$$

\subsubsection{Energy-Causality Constraint for the BD's Transmission.} This constraint is to ensure that each BD only uses its harvested energy to support the energy consumption for the passive and active communications so that the energy early stored in its battery is saved. Following reference [6], a fixed power consumption model is considered for BackCom, where the power consumption for the $k$-th $\mathrm{BD}$ is fixed as a constant, denoted by $P_{b, k}$. Then, the total energy consumption for BackCom at the $k$-th $\mathrm{BD}$ is given by $P_{b, k} t_{k}$. Let $p_{a, k}$ denote the constant circuit power consumption for active communications at the $k$-th $\mathrm{BD}$. Then, the total energy consumption for active communications at the $k$-th $\mathrm{BD}$ can be computed as $p_{k} \tau_{k}+p_{a, k} \tau_{k}$. On this basis, the energycausality constraint for the $k$-th $\mathrm{BD}$ can be represented as

$$
\begin{aligned}
P_{b, k} t_{k} & +p_{k} \tau_{k}+p_{a, k} \tau_{k} \leq E_{k} \\
& =\left(\beta-t_{k}\right) \phi_{k}\left[P_{0} g_{k}\right]+t_{k} \phi_{k}\left[P_{0} g_{k}\left(1-\alpha_{k}\right)\right], \quad \forall k
\end{aligned}
$$

3.1.4. The Minimum Required SINR Constraint for Decoding $x_{p}$. This constraint is to ensure that the BR can decode $x_{p}$ successfully. Without this constraint, the BR may not decode $x_{b, k}$, leading to $C_{b, k}=0$. Thus, this constraint is necessary for the considered network. Accordingly, the minimum required SINR constraint for decoding $x_{p}$ is given by

$$
\gamma_{b, k}^{p} \geq \gamma_{t h}, \forall k, \Leftrightarrow \frac{P_{0} h_{k}}{\alpha_{k} P_{0} g_{k} f_{k}+\sigma^{2}} \geq \gamma_{t h}, \quad \forall k .
$$

3.1.5. Transmit Power Constraint for the PT. Let $P_{\max }$ express the maximum allowed transmit power for the PT. Then, the transmit power constraint for the PT is given by

$$
0 \leq P_{0} \leq P_{\max }
$$

3.1.6. Throughput Fairness Optimization Problem. Based on (16), (18), (19), (20), and (21), the throughput fairness optimization problem is formulated as

$$
\begin{aligned}
& \mathbf{P}_{1}: \max _{P_{0}, t_{e}, \beta,\left\{t_{k}\right\}_{k=1}^{K},\left\{\alpha_{k}\right\}_{k=1}^{K}\left\{p_{k}\right\}_{k=1}^{K},\left\{\tau_{k}\right\}_{k=1}^{K}} \min _{k} C_{\text {tot }}^{k} \\
& \text { s.t. C1: }(18), \\
& \text { C2: }(19), \\
& \text { C3: }(20), \\
& \text { C4: }(21), \\
& \text { C5: } t_{e}+\sum_{k=1}^{K} t_{k} \leq \beta, \sum_{k=1}^{K} \tau_{k} \leq T-\beta, \\
& 0 \leq \beta \leq T, t_{e}, t_{k}, \tau_{k} \geq 0, \quad \forall k, \\
& \text { C6: } 0 \leq \alpha_{k} \leq 1, \quad \forall k,
\end{aligned}
$$

where C1 expresses the QoS constraint for the primary transmission, $\mathrm{C} 2$ denotes the energy-causality constraint for each BD, C3 is the minimum required SINR for decoding $x_{p}$, $\mathrm{C} 4$ constrains the maximum transmit power of the PT, C5 is the constraint for the EH time, the BackCom time, etc., and C6 is the constraint for the power reflection coefficient of each BD.

As for $\mathbf{P}_{1}$, it is highly nonconvex and hard to solve. The reasons are as follows: firstly, the min function is involved in the objective function, which makes the objective function more complex and difficult to handle. Secondly, both the cochannel interference and the remaining part due to imperfect SIC exist, bringing the difference of convex (DC) structures in both the objective function and constraint $\mathrm{C} 1$ and leading to highly nonconvex objective function and C1. Thirdly, the use of the nonlinear EH model makes C2 more complex, which brings a new challenge to solve $\mathbf{P}_{1}$. Finally, except the above difficulties, several coupled relationships among multiple optimization variables exist, i.e., $P_{t}, t_{k}$, and $\alpha_{k}$, leading to the nonconvex objective function and constraints, e.g., C1, C2, etc.

3.2. Solution. This subsection is provided to solve $\mathbf{P}_{1}$ efficiently. Firstly, in order to remove the min function in the objective function and simplify the objective function further, we introduce an auxiliary variable $\lambda$ into $\mathbf{P}_{1}$ by letting $\lambda=\min _{k} C_{\text {tot }}^{k}$. Then, we can rewrite $\mathbf{P}_{1}$ as

$$
\begin{aligned}
& \mathbf{P}_{2}: \max _{P_{0}, t_{e}, \beta, \lambda,\left\{t_{k}\right\}_{k=1}^{K},\left\{\alpha_{k}\right\}_{k=1}^{K}\left\{p_{k}\right\}_{k=1}^{K},\left\{\tau_{k}\right\}_{k=1}^{K}} \lambda \\
& \text { s.t. C1 - C6, } \\
& \text { C7: } C_{b, k}+C_{a, k} \geq \lambda .
\end{aligned}
$$

As for $\mathbf{P}_{2}$, it is still nonconvex since the DC structures, the nonlinear EH model, and the coupled relationships still exist. In order to handle the DC structure in $C_{b, k}$, we provide the following proposition to determine the optimal transmit power of the PT $P_{0}^{*}$.

Proposition 1. In the considered network, the minimum throughput of each BD is maximized when the PT transmit its information with its maximum allowed transmission power, e.g., $P_{0}^{*}=P_{\max }$. 
Proof. Here, we prove Proposition 1 by means of contradiction. We assume that $\left\{P_{0}^{*}, t_{e}^{*}, \beta^{*}, \lambda^{*},\left\{t_{k}^{*}\right\}_{k=1}^{K},\left\{\alpha_{k}^{*}\right\}_{k=1}^{K}\right.$, $\left.\left\{p_{k}^{*}\right\}_{k=1}^{K},\left\{\tau_{k}^{*}\right\}_{k=1}^{K}\right\}$ is the optimal solution to $\mathbf{P}_{2}$, where both $P_{0}^{*}<P_{\max }$ and $\lambda^{*}=\min t_{k}^{*} W \log _{2}\left(1+\left(\left(\xi \alpha_{k}^{*} P_{0}^{*} g_{k} f_{k}\right) /\right.\right.$ $\left.\left.\left(\epsilon P_{0}^{*} h_{k}+\sigma^{2}\right)\right)\right)+\tau_{k}^{*} W \log _{2}^{k}\left(1+\left(p_{k}^{*} f_{k} / \sigma^{2}\right)\right)$ hold. Then, another solution can be constructed, given by $P_{0}^{+}=P_{\max }, t_{e}^{+}=$ $t_{e}^{*}, \beta^{+}=\beta^{*}, t_{k}^{+}=t_{k}^{*}, \alpha_{k}^{+}=\alpha_{k}^{*}, p_{k}^{+}=p_{k}^{*}, \tau_{k}^{+}=\tau_{k}^{*}$. Obviously, the constructed solution is a feasible solution which satisfies all the constraints of $\mathbf{P}_{2}$. Accordingly, we can compute $\lambda^{+}$ as $\min _{k} t_{k}^{+} W \log _{2}\left(1+\left(\left(\xi \alpha_{k}^{+} P_{0}^{+} g_{k} f_{k}\right) /\left(\epsilon P_{0}^{+} h_{k}+\sigma^{2}\right)\right)\right)+\tau_{k}^{+} W$ $\log _{2}\left(1+\left(\left(p_{k}^{+} f_{k}\right) / \sigma^{2}\right)\right)$. Since $P_{0}^{+}=P_{\max }>P_{0}^{*}$ holds, we can prove that $\lambda^{+}>\lambda^{*}$ is satisfied. The reasons are as follows: let $F_{k}\left(P_{0}\right)=t_{k} W \log _{2}\left(1+\left(\left(\xi \alpha_{k} P_{0} g_{k} f_{k}\right) /\left(\epsilon P_{0} h_{k}+\sigma^{2}\right)\right)\right)$.

Then, the first-order derivative of $F_{k}\left(P_{0}\right)$ with respect to $P_{0}$ is given by

$$
\frac{\partial F_{k}\left(P_{0}\right)}{\partial P_{0}}=\frac{t_{k} W \xi \alpha_{k} f_{k} g_{k} \sigma^{2}}{\left(\epsilon P_{0} h_{k}+\sigma^{2}\right)\left(\epsilon P_{0} h_{k}+\sigma^{2}+\xi \alpha_{k} f_{k} g_{k} P_{0}\right) \operatorname{In} 2} .
$$

Since $\left(\partial F_{k}\left(P_{0}\right) / \partial P_{0}\right)>0$ always holds, $F_{k}\left(P_{0}\right)$ will increase with the increasing of $P_{0}$. That is to say, $\lambda^{+}>\lambda^{*}$ holds, which contradicts the assumption that $P_{0}^{*}<P_{\max }$. Therefore, $P_{0}^{*}=P_{\max }$ holds when the minimum throughput of each BD is maximized for the considered network. Hence, the proof is complete.

Based on Proposition 1, we substitute $P_{0}=P_{\max }$ into $\mathbf{P}_{2}$ and reformulate $\mathbf{P}_{2}$ as

$$
\begin{aligned}
& \mathbf{P}_{3}: \max _{t_{e}, \beta, \lambda,\left\{t_{k}\right\}_{k=1}^{K},\left\{\alpha_{k}\right\}_{k=1}^{K},\left\{p_{k}\right\}_{k=1}^{K},\left\{\tau_{k}\right\}_{k=1}^{K}} \lambda \\
& \text { s.t. } \mathrm{C} 1^{\prime}: \sum_{k=1}^{K} t_{k} W \log _{2}\left(1+\frac{P_{\max } h_{p}}{\alpha_{k} P_{\max } g_{k} f_{p, k}+\sigma^{2}}\right) \\
& +t_{e} W \log _{2}\left(1+\frac{P_{\max } h_{p}}{\sigma^{2}}\right) \geq C_{\min } \\
& \mathrm{C} 2^{\prime}: P_{\mathrm{b}, k} t_{k}+p_{k} \tau_{k}+p_{a, k} \tau_{k} \leq\left(\beta-t_{k}\right) \phi_{k}\left[P_{\max } g_{k}\right] \\
& +t_{k} \phi_{k}\left[P_{\max } g_{k}\left(1-\alpha_{k}\right)\right], \forall k, \\
& \mathrm{C}^{\prime}: 0 \leq \alpha_{k} \leq \min \left(\frac{P_{\max } h_{k}-\gamma_{\mathrm{th}} \sigma^{2}}{P_{\max } f_{k} g_{k} \gamma_{t h}}, 1\right), \forall k, \mathrm{C} 5, \\
& \mathrm{C}^{\prime}: t_{k} W \log _{2}\left(1+\frac{\xi \alpha_{k} P_{\max } g_{k} f_{k}}{\epsilon P_{\max } h_{k}+\sigma^{2}}\right) \\
& +\tau_{k} W \log _{2}\left(1+\frac{p_{k} f_{k}}{\sigma^{2}}\right) \geq \lambda, \forall k
\end{aligned}
$$

where $\mathrm{C}^{\prime}$ is the combination of $\mathrm{C} 3$ and C6.

It can be observed from $\mathbf{P}_{3}$ that $\mathbf{P}_{3}$ is still a nonconvex problem. To deal with the coupled relationships among different variables, i.e., $\alpha_{k}$ and $t_{k}, p_{k}$ and $\tau_{k}$, the following auxiliary variables, $x_{k}=\alpha_{k} t_{k}, y_{k}=p_{k} \tau_{k}, \forall k$, are introduced in $\mathbf{P}_{3}$ to replace variables $\alpha_{k}, p_{k}, \forall k$. Then, $\mathbf{P}_{3}$ is reformulated as

$$
\begin{aligned}
& \mathbf{P}_{4}: \max _{t_{e}, \beta, \lambda,\left\{t_{k}\right\}_{k=1}^{K},\left\{x_{k}\right\}_{k=1}^{K},\left\{y_{k}\right\}_{k=1}^{K},\left\{\tau_{k}\right\}_{k=1}^{K}} \lambda \\
& \text { s.t. } C 1^{\prime \prime}: \sum_{k=1}^{K} t_{k} W \log _{2}\left(1+\frac{P_{\max } h_{p} t_{k}}{x_{k} P_{\max } g_{k} f_{p, k}+t_{k} \sigma^{2}}\right) \\
& +t_{e} W \log _{2}\left(1+\frac{P_{\max } h_{p}}{\sigma^{2}}\right) \geq C_{\text {min }} \\
& \mathrm{C} 2^{\prime \prime}: P_{\mathrm{b}, k} t_{k}+y_{k}+p_{a, k} \tau_{k} \leq\left(\beta-t_{k}\right) \phi_{k}\left[P_{\max } g_{k}\right] \\
& +t_{k} \phi_{k}\left[\frac{P_{\max } g_{k}\left(t_{k}-x_{k}\right)}{t_{k}}\right], \forall k \text {, } \\
& \mathrm{C}^{\prime \prime}: 0 \leq x_{k} \leq t_{k} \times \min \left(\frac{P_{\max } h_{k}-\gamma_{\mathrm{th}} \sigma^{2}}{P_{\max } f_{k} g_{k} \gamma_{t h}}, 1\right), \forall k, \mathrm{C} 5 \text {, } \\
& \mathrm{C}^{\prime \prime}: t_{k} W \log _{2}\left(1+\frac{\xi x_{k} P_{\max } g_{k} f_{k}}{t_{k}\left(\epsilon P_{\max } h_{k}+\sigma^{2}\right)}\right) \\
& +\tau_{k} W \log _{2}\left(1+\frac{y_{k} f_{k}}{\tau_{k} \sigma^{2}}\right) \geq \lambda, \forall k,
\end{aligned}
$$

where $\alpha_{k}=\left(x_{k} / t_{k}\right), p_{k}=\left(y_{k} / \tau_{k}\right), \forall k$.

Proposition 2. As for $\mathbf{P}_{4}$, the objective function and all the constraints except $C 1^{\prime \prime}$ are convex.

Proof. It can be observed that the objective function and the constraint $\mathrm{C}^{\prime \prime}$ are linear, which are also convex. For the constraint $\mathrm{C7}^{\prime \prime}$, using the fact that the perspective function can preserve convexity, we can conclude that the convexities of functions $t_{k} W \log _{2}\left(1+\left(\left(\xi x_{k} P_{\max } g_{k} f_{k}\right) /\left(t_{k}\left(\epsilon P_{\max } h_{k}+\right.\right.\right.\right.$ $\left.\left.\left.\left.\sigma^{2}\right)\right)\right)\right)$ and $\tau_{k} W \log _{2}\left(1+\left(y_{k} f_{k} / \tau_{k} \sigma^{2}\right)\right)$ are the same as those of $W \log _{2}\left(1+\left(\left(\xi x_{k} P_{\max } g_{k} f_{k}\right) /\left(\epsilon P_{\max } h_{k}+\sigma^{2}\right)\right)\right)$ and $W$ $\log _{2}\left(1+\left(y_{k} f_{k} / \sigma^{2}\right)\right)$. Since both $W \log _{2}\left(1+\left(\left(\xi x_{k} P_{\max }\right.\right.\right.$ $\left.\left.\left.g_{k} f_{k}\right) /\left(\epsilon P_{\max } h_{k}+\sigma^{2}\right)\right)\right)$ and $W \log _{2}\left(1+\left(y_{k} f_{k} / \sigma^{2}\right)\right)$ are concave functions, $t_{k} W \log _{2}\left(1+\left(\left(\xi x_{k} P_{\max } g_{k} f_{k}\right) /\left(t_{k}\right.\right.\right.$ $\left.\left.\left.\left(\epsilon P_{\max } h_{k}+\sigma^{2}\right)\right)\right)\right)$ and $\tau_{k} W \log _{2}\left(1+\left(y_{k} f_{k} / \tau_{k} \sigma^{2}\right)\right)$ are also concave. Thus, $\mathrm{C}^{\prime \prime}$ is a convex constraint.

For the constraint $\mathrm{C} 2{ }^{\prime \prime}$, its convexity depends on the convexity of $t_{k} \phi_{k}\left[\left(P_{\max } g_{k}\left(t_{k}-x_{k}\right)\right) /\left(t_{k}\right)\right]$. According to the perspective function, the convexity of $t_{k} \phi_{k}\left[\left(P_{\max } g_{k}\left(t_{k}-\right.\right.\right.$ $\left.\left.\left.x_{k}\right)\right) /\left(t_{k}\right)\right]$ is the same as that of $\phi_{k}\left[P_{\max } g_{k}\left(1-x_{k}\right)\right]$. As pointed out in [6], $\phi_{k}\left[P_{\max } g_{k}\left(1-x_{k}\right)\right]$ can be proved to be concave by using the properties of practical $\mathrm{EH}$ circuits. Thus, the constraint $\mathrm{C} 2{ }^{\prime \prime}$ is convex.

Based on the above analysis, Proposition 2 is achieved, and the proof is complete.

We note that the nonconvexity of $\mathrm{C}^{\prime \prime}$ is due to the existence of the DC structure, i.e., $\sum_{k=1}^{K} t_{k} W \log _{2}(1+$ $\left.\left(\left(P_{\max } h_{p} t_{k}\right) /\left(x_{k} P_{\max } g_{k} f_{p, k}+t_{k} \sigma^{2}\right)\right)\right)$. To address this problem, we use the SCA method to deal with the nonconvexity of $\mathrm{Cl}^{\prime \prime}$. Specifically, we first replace $\sum_{k=1}^{K}$ $t_{k} W \log _{2}\left(1+\left(P_{\max } h_{p} t_{k}\right) /\left(x_{k} P_{\max } g_{k} f_{p, k}+t_{k} \sigma^{2}\right)\right)$ with its first-order Taylor expression so that $\mathrm{Cl}^{\prime \prime}$ can be turned into 
a linear constraint, which is always a convex constraint. Then, we can use the existing convex tools to solve the convex subproblem by changing $\mathbf{P}_{4}$ with the first-order Taylor expression. Finally, an efficient iterative algorithm is proposed based on the SCA method to solve $\mathbf{P}_{4}$, where the above subproblem is solved in each iteration.
Let $G_{k}\left[\alpha_{k}\right]$ denote $t_{k} W \log _{2}\left(1+\left(\left(P_{\max } h_{p}\right) /\left(\alpha_{k} P_{\max }\right.\right.\right.$ $\left.\left.\left.g_{k} f_{p, k}+\sigma^{2}\right)\right)\right)$. Then, $\sum_{k=1}^{K} t_{k} W \log _{2}\left(1+\left(\left(P_{\max } h_{p} t_{k}\right) /\left(x_{k}\right.\right.\right.$ $\left.\left.\left.P_{\max } g_{k} f_{p, k}+t_{k} \sigma^{2}\right)\right)\right)$ can be denoted by $\sum_{k=1}^{K} G_{k}\left[\alpha_{k}\right]$. By taking the first-order derivative of $G_{k}\left[\alpha_{k}\right]$ with respect to $\alpha_{k}$, we have

$$
\frac{\partial G_{k}\left[\alpha_{k}\right]}{\partial \alpha_{k}}=\frac{-P_{\max ^{2}} h_{p} g_{k} f_{p, k} W t_{k}}{\left(\alpha_{k} P_{\max } g_{k} f_{p, k}+\sigma^{2}+P_{\max } h_{p}\right)\left(\alpha_{k} P_{\max } g_{k} f_{p, k}+\sigma^{2}\right) \operatorname{In} 2} .
$$

Accordingly, the first-order Taylor expression of $G_{k}\left[\alpha_{k}\right]$ on a given value $\alpha_{k}^{0}$ can approximate $G_{k}\left[\alpha_{k}\right]$ as

$$
\begin{aligned}
G_{k}\left[\alpha_{k}\right] & \approx \frac{\partial G_{k}\left(\alpha_{k}^{0}\right)}{\partial \alpha_{k}^{0}}\left(\alpha_{k}-\alpha_{k}^{0}\right)+G_{k}\left[\alpha_{k}^{0}\right] \\
& =\frac{-P_{\max ^{2}} h_{p} g_{k} f_{p, k} W t_{k}\left(\alpha_{k}-\alpha_{k}^{0}\right)}{\left(\alpha_{k}^{0} P_{\max } g_{k} f_{p, k}+\sigma^{2}+P_{\max } h_{p}\right)\left(\alpha_{k}^{0} P_{\max } g_{k} f_{p, k}+\sigma^{2}\right) \operatorname{In} 2}+G_{k}\left[\alpha_{k}^{0}\right] \\
& =\frac{-P_{\max ^{2}} h_{p} g_{k} f_{p, k} W\left(x_{k}-\alpha_{k}^{0} t_{k}\right)}{\left(\alpha_{k}^{0} P_{\max } g_{k} f_{p, k}+\sigma^{2}+P_{\max } h_{p}\right)\left(\alpha_{k}^{0} P_{\max } g_{k} f_{p, k}+\sigma^{2}\right) \operatorname{In} 2}+G_{k}\left[\alpha_{k}^{0}\right],
\end{aligned}
$$

where $\alpha_{k}^{0}$ will be updated in each iteration based on $\alpha_{k}$ obtained in the previous iteration.
Based on (28), the following subproblem can be obtained from $\mathbf{P}_{4}$, given by

$$
\begin{aligned}
& \mathbf{P}_{5}: \max _{t_{e}, \beta, \lambda,\left\{t_{k}\right\}_{k=1}^{K},\left\{x_{k}\right\}_{k=1}^{K},\left\{y_{k}\right\}_{k=1}^{K},\left\{\tau_{k}\right\}_{k=1}^{K}} \lambda \\
& \text { s.t. C1 }{ }^{\prime \prime \prime}: \sum_{k=1}^{K} \frac{-P_{\max ^{2}} h_{p} g_{k} f_{p, k} W\left(x_{k}-\alpha_{k}^{0} t_{k}\right)}{\left(\alpha_{k}^{0} P_{\max } g_{k} f_{p, k}+\sigma^{2}+P_{\max } h_{p}\right)\left(\alpha_{k}^{0} P_{\max } g_{k} f_{p, k}+\sigma^{2}\right)} \\
& \quad \times \frac{1}{\operatorname{In} 2}+G_{k}\left[\alpha_{k}^{0}\right]+t_{e} W \log _{2}\left(1+\frac{P_{\max } h_{p}}{\sigma^{2}}\right) \geq C_{\min }, \\
& \text { C2 }^{\prime \prime}, \mathrm{C} 3^{\prime \prime}, C 7^{\prime \prime} .
\end{aligned}
$$

Proposition 3. $\mathbf{P}_{5}$ is convex and can be efficiently solved by using the existing convex tools.

Proof. It can be observed that $\mathrm{Cl}^{\prime \prime \prime}$ is a linear constraint, which is obviously convex. Combining with Proposition 2, $\mathbf{P}_{5}$ is convex, which can be efficiently solved by using the existing convex optimization tools.

3.3. Iterative Algorithm. In this subsection, we propose an efficient iterative algorithm to solve $\mathbf{P}_{4}$, as shown in Algorithm 1. In particular, the subproblem $\mathbf{P}_{5}$ is optimally solved under given $\alpha_{k}^{0}, \forall k$ in each iteration, and the values of $\alpha_{k}^{0}, \forall k$ are updated based on the optimal power reflection coefficients $\alpha_{k}^{*}, \forall k$ obtained in the previous iteration. We note that for the first iteration, the values of $\alpha_{k}^{0}, \forall k$ are predefined. The optimal solution to $\mathbf{P}_{4}$ is achieved when the algorithm converges, namely, the stop condition $\left|\alpha_{k}^{*}-\alpha_{k}^{0}\right| \leq \varepsilon$ with the maximum tolerance $\varepsilon$ is satisfied.

We provide the analysis of the computational complexity of Algorithm 1 as follows: we assume that the interior point method is applied to solve $\mathbf{P}_{5}$ with given $\alpha_{k}^{0}, \forall k$. Let $m_{1}$ and $N_{u}$ denote the number of the inequality constraints of $\mathbf{P}_{5}$ and the number of iterations for Algorithm 1, respectively. Then, the computational complexity of Algorithm 1 can be calculated as $N_{u} O\left(\sqrt{m_{1}} \log \left(m_{1}\right)\right)$ [16]. 
Algorithm 1: An efficient iterative algorithm for solving $\mathbf{P}_{4}$.

(1) Set the maximum tolerance $\varepsilon$ and the maximum number of iterations $I_{\max }$;

(2) Set the iteration index $i=1$ and the initial given values $\alpha_{k}^{0}, \forall k$;

(3) repeat

(4) Solve $\mathbf{P}_{5}$ with given $\alpha_{k}^{0}, \forall k$ via CVX, to obtain its optimal solution, denoted by $\left\{t_{e}^{*}, \beta^{*}, \lambda^{*},\left\{t_{k}^{*}\right\}_{k=1}^{K},\left\{x_{k}^{*}\right\}_{k=1}^{K},\left\{\tau_{k}^{*}\right\}_{k=1}^{K},\left\{y_{k}^{*}\right\}_{k=1}^{K}\right\}$;

(5) Compute $\alpha_{k}^{*}$ as $\left(x_{k}^{*} / t_{k}^{*}\right), \forall k$ and $p_{k}^{*}$ as $\left(y_{k}^{*} / \tau_{k}^{*}\right), \forall k$;

(6) if $\left|\alpha_{k}^{*}-\alpha_{k}^{0}\right| \leq \varepsilon$ then

(7) $\quad$ Set Flag $=1$;

(8) else

(9) Set Flag $=0$ and $i=i+1$;

(10) Update $\alpha_{k}^{0}$ as $\alpha_{k}^{0}=\alpha_{k}^{*}, \forall k$;

(11) end if

(12) until Flag $=1$ or $i=I_{\max }$.

(13) Output the optimal solution to $\mathbf{P}_{4}$ as $\left\{t_{e}^{*}, \beta^{*}, \lambda^{*},\left\{t_{k}^{*}\right\}_{k=1}^{K},\left\{\alpha_{k}^{*}\right\}_{k=1}^{K},\left\{\tau_{k}^{*}\right\}_{k=1}^{K},\left\{p_{k}^{*}\right\}_{k=1}^{K}\right\}$.

Algorithm 1

\section{Numerical Results}

In this section, we use computer simulations to verify the superiority of our proposed resource allocation scheme and the effectiveness of the proposed algorithm. Unless otherwise specified, the basic simulation parameters are set, as shown in Table $1[11,13]$. According to [14], we set $a_{k}=2.463, d_{k}=1.635$, and $v_{k}=0.826, \forall k$ to characterize the used nonlinear EH model clearly. Besides, all the channels are set as follows: Following the standard channel fading model, the channel gain is the product of the small-scale fading and the large-scale fading. Let $g_{k}^{\prime}, f_{k}^{\prime}, h_{k}^{\prime}, f_{p, k}{ }^{\prime}$, and $h_{p}^{\prime}$ denote the small-scale fadings of the PT-the $k$-th BD link, the $k$-th $\mathrm{BD}$-its receiver link, the $\mathrm{PT}$-the $k$-th $\mathrm{BR}$ link, the $k$-th BD-the PR link, and the PT-PR link, respectively. We denote $D_{1, k}, D_{2, k}, D_{3, k}, D_{4, k}$, and $D_{p}$ as the distances of the PT-the $k$-th $\mathrm{BD}$ link, the $k$-th $\mathrm{BD}$-its receiver link, the PT-the $k$-th $\mathrm{BR}$ link, the $k$-th $\mathrm{BD}$-the PR link, and the PT-PR link, respectively. Then, we have $g_{k}=g_{k}^{\prime} D_{1, k}^{-\zeta}, f_{k}=f_{k}^{\prime} D_{2, k}^{-\zeta}$, $h_{k}=h_{k}^{\prime} D_{3, k}^{-\zeta}, f_{p, k}=f_{p, k}^{\prime} D_{4, k}^{-\zeta}$, and $h_{p}=h_{p}^{\prime} D_{p}^{-\zeta}$, where $\zeta \mathrm{de}-$ notes the path loss exponent. Here, we set $\zeta=3, D_{1,1}=11$ $m, D_{1,2}=12 m, D_{1,3}=15 m, D_{1,4}=14 m, D_{2,1}=40 m$, $D_{2,2}=35 m, D_{2,3}=32 m, D_{2,4}=35 m, D_{3,1}=35 \mathrm{~m}$, $D_{3,2}=32 m, D_{3,3}=40 m, D_{3,4}=35 m, D_{4,1}=50 m$, $D_{4,2}=55 \mathrm{~m}, D_{4,3}=53 \mathrm{~m}, D_{4,4}=52 \mathrm{~m}$, and $D_{p}=30 \mathrm{~m}$.

In order to illustrate the superiority of the proposed scheme, we compare the performance under the proposed scheme with that of the following benchmark schemes: backscatter-assisted cognitive networks and wireless powered cognitive networks. For the backscatter-assisted cognitive networks, each BD only backscatters its information to its receiver, while for the wireless powered cognitive networks, each BD first harvests energy from the PT's signals when PT is busy and then uses its harvested energy to transmit its information to its receiver. We note that both backscatter-assisted cognitive networks and wireless powered cognitive networks can be regarded as special cases for the considered network and can be obtained after a few changes on the considered network. For example, let $p_{k}=0$ and $\tau_{k}=0$ and the backscatter-assisted cognitive networks can be achieved. That is to say, the proposed algorithm can also be used to obtain the optimal schemes under backscatter-assisted cognitive networks and wireless powered cognitive networks.

Figure 3 shows the convergence of Algorithm 1, where different settings of $P_{\max }$ are considered. Here, we set $P_{\max }$ as $0.5 \mathrm{~W}, 0.8 \mathrm{~W}$, and $1 \mathrm{~W}$, respectively. It can be seen that Algorithm 1 can always converge to a certain value after only a few iterations, i.e., 2 iterations, which illustrates the convergence of Algorithm 1 and shows that Algorithm 1 is computationally efficient. Besides, we can also see that with a larger $P_{\max }$, the minimum throughput achieved by BDs also increases. This is because the optimal transmit power of the PT is determined by $P_{\max }$ and a larger $P_{0}$ brings a higher throughput.

Figure 4 shows the minimum throughput achieved by BDs versus the maximum allowed transmit power of the PT $P_{\text {max }}$, where $P_{\text {max }}$ is varied from $0.5 \mathrm{~W}$ to $2.5 \mathrm{~W}$. In order to demonstrate the superiority of the proposed scheme, we compare the performance under the proposed scheme with that of backscatter-assisted cognitive networks and wireless powered cognitive networks. It can be seen that the minimum throughput achieved by BDs increases with the increasing of $P_{\max }$ since a larger $P_{\max }$ means a higher $P_{0}$, which brings a higher throughput for each $\mathrm{BD}$. Besides, comparing with backscatter-assisted cognitive networks and wireless powered cognitive networks, we can also find that the proposed scheme outperforms the other schemes as the proposed scheme has more flexibility to use resources efficiently, which also illustrates the advantages of the proposed scheme.

Figure 5 shows the minimum throughput among BDs versus the minimum required throughput for the $\mathrm{PT}, C_{\min }$, where $C_{\min }$ is ranged from 1 Mbyte to $1.5 \mathrm{Mbytes}$. From this figure, it can be seen that the minimum throughput achieved by BDs decreases with the increasing of $C_{\min }$, as a larger $C_{\min }$ means a higher QoS requirement for the PT's transmission, and more resources will be allocated to the PT, leading to a reduction to the throughput achieved by each BD. By comparisons, we can see that the proposed scheme can achieve higher throughput than the other schemes, verifying the advantages of the proposed scheme. 
TABle 1: Basic simulation settings.

\begin{tabular}{lcc}
\hline Parameters & Notation & Value \\
\hline The entire time block & $T$ & $1 \mathrm{~s}$ \\
The system bandwidth & $P_{b, k}$ & $100 \mathrm{kHz}$ \\
The constant circuit power consumption for BackCom at the $k$-th BD & $p_{a, k}$ & $10 \mu \mathrm{W}$ \\
The constant circuit power consumption for active communications at the $k$-th BD & $\xi$ & $1 \mathrm{~mW}$ \\
The maximum transmit power at the PT & $\sigma^{2}$ & $K$ \\
The performance gap reflecting the real modulation for BackCom & $K$ & $-15 \mathrm{~dB}$ \\
The noise power & $C_{\text {min }}$ & 4 \\
The number of BDs & $\gamma_{t h}$ & $1000 \mathrm{kbytes}$ \\
The minimum required throughput of the PT & 20 \\
The threshold required for decoding $x_{p}$ &
\end{tabular}

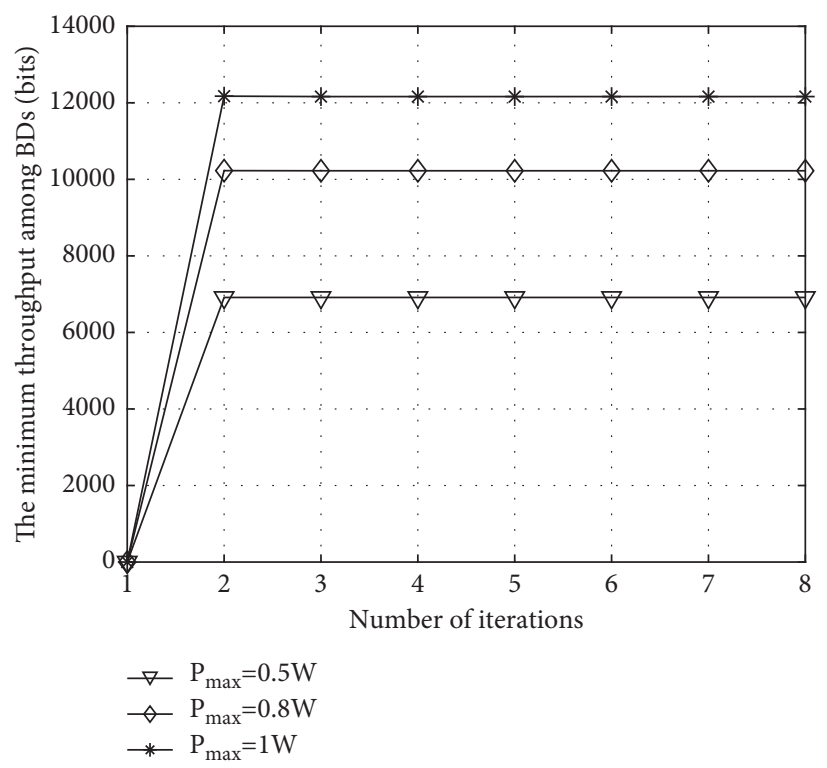

Figure 3: The convergence of Algorithm 1 under different settings of $P_{\max }$.

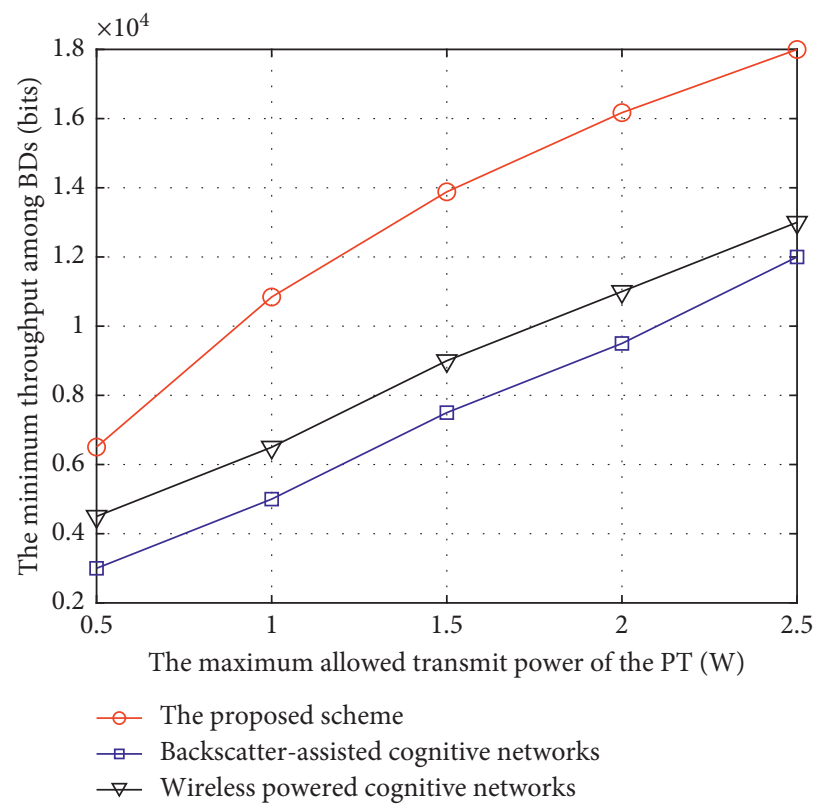

FIgUre 4: The minimum throughput among BDs versus the maximum allowed transmit power of the PT. 


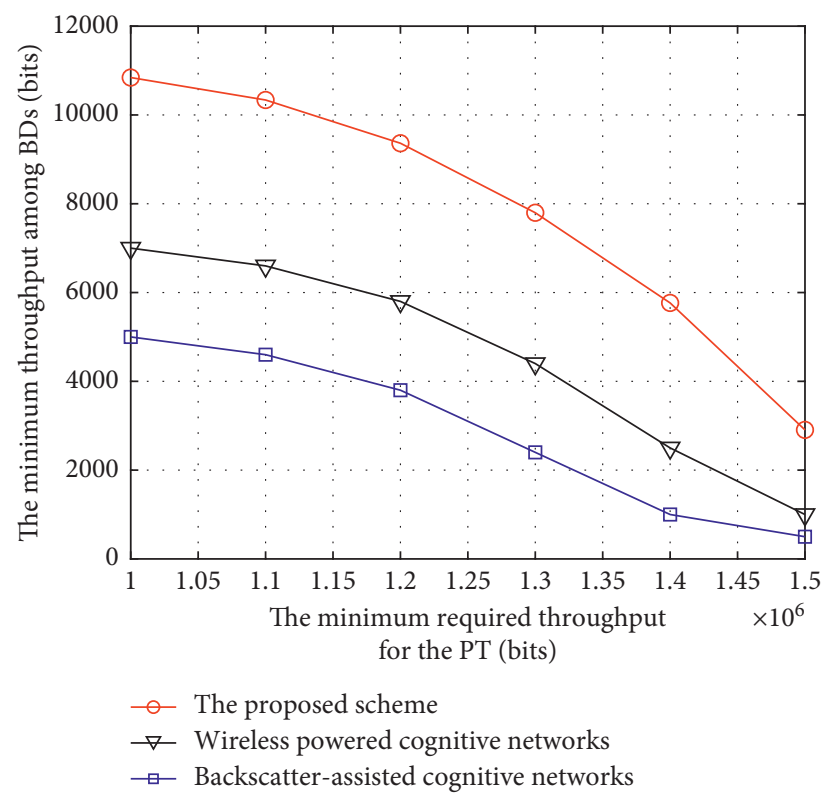

FIgURE 5: The minimum throughput among BDs versus the minimum required throughput for the PT.

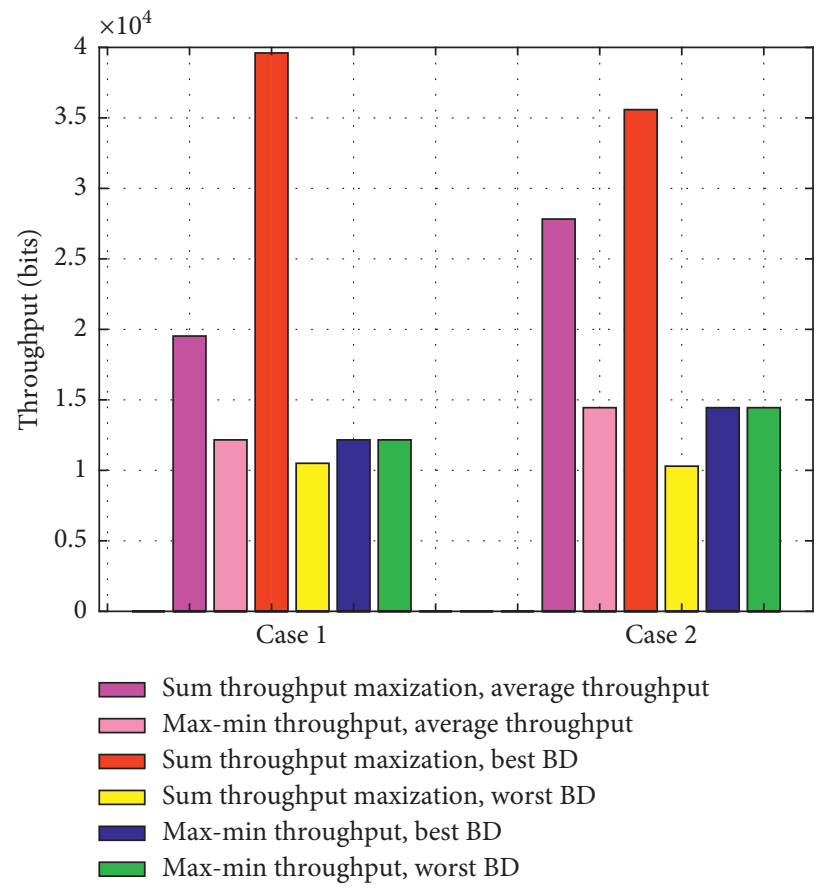

FIGURE 6: Fairness comparison.

Figure 6 compares the $\mathrm{BD}$ fairness achieved by the proposed scheme, denoted by max-min throughput, and the sum throughput maximization scheme, denoted by sum throughput maximization, under two cases, where the settings of the channels are different. It can be seen that there exists a tradeoff between the sum throughput maximization and the max-min throughput. Specifically, the proposed scheme can greatly improve the fairness among BDs while the average throughput among all $\mathrm{BDs}$ is reduced. This is because, for the proposed scheme, more resources will be allocated to the $\mathrm{BD}$ with a worst channel condition for good throughput fairness, while for the sum throughput 
maximization, more resources will be allocated to the $\mathrm{BD}$ with a better channel condition for achieving the maximum throughput of all BDs.

\section{Conclusions}

In this paper, we have studied the throughput fairness for the wireless powered cognitive hybrid active-passive communication network while considering a nonlinear EH model. In particular, we have formulated an optimization problem to maximize the minimum throughput that achieved all BDs by jointly optimizing the transmit power and time of the PT, the BDs' time sharing among $\mathrm{EH}$, BackCom and active communications, and the power reflection coefficient and transmit power of each $\mathrm{BD}$ subject to the QoS, energy causality, transmit power constraints, etc. In order to solve this problem, the optimal transmit power of the PT was firstly achieved by means of contradiction, and then an efficient iterative algorithm was developed to obtain the optimal solutions. Simulation results verified the quick convergence of the proposed algorithm and demonstrated the superiority of the proposed scheme in terms of throughput fairness.

\section{Data Availability}

The simulation data used to support the findings of this study are included within the article. The MATLAB code used to support the findings of this study are available from the corresponding author upon request.

\section{Conflicts of Interest}

The authors declare that they have no conflicts of interest.

\section{Acknowledgments}

This work was supported by the scholarship from China Scholarship Council (no. 201708230301), the Science Foundation of Heilongjiang Province for the Excellent Youth (no. YQ2019F014), the Science Talent Support Program of Heilongjiang Bayi Agricultural University (no. ZRCQC201807), and the Scientific Research Foundation for Doctor of Heilongjiang Bayi Agricultural University (no. XDB2015-28).

\section{References}

[1] A. Al-Fuqaha, M. Guizani, M. Mohammadi, M. Aledhari, and M. Ayyash, "Internet of things: a survey on enabling technologies, protocols, and applications," IEEE Communications Surveys \& Tutorials, vol. 17, no. 4, pp. 2347-2376, 2015.

[2] J. M. Peha, "Sharing spectrum through spectrum policy reform and cognitive radio," Proceedings of the IEEE, vol. 97, no. 4, pp. 708-719, 2009.

[3] Y. Xu, H. Sun, and Y. Ye, "Distributed resource allocation for swipt-based cognitive ad-hoc networks," IEEE Transactions on Cognitive Communications and Networking, vol. 7, no. 4, pp. 1320-1332, 2021.

[4] A. Costanzo, D. Masotti, G. Paolini, and D. Schreurs, "Evolution of SWIPT for the iot world: near- and far-field solutions for simultaneous wireless information and power transfer," IEEE Microwave Magazine, vol. 22, no. 12, pp. 48-59, 2021.

[5] H. Yang, Y. Ye, X. Chu, and S. Sun, "Energy efficiency maximization for UAV-enabled hybrid backscatter-harvestthen-transmit communications," IEEE Transactions on Wireless Communications, p. 1, 2021, https://ieeexplore.ieee. org/document/9562293.

[6] Y. Ye, L. Shi, X. Chu, and G. Lu, "Throughput fairness guarantee in wireless powered backscatter communications with HTT," IEEE Wireless Communications Letters, vol. 10, no. 3, pp. 449-453, 2021.

[7] T. D. Tran and L. B. Le, "Hybrid backscatter and underlay transmissions in RF-powered cognitive radio networks," in Proceedings of the 2019 26th International Conference on Telecommunications (ICT), pp. 11-15, Hanoi, Vietnam, April 2019.

[8] X. Lu, D. Niyato, H. Jiang, D. I. Kim, Y. Xiao, and Z. Han, "Ambient backscatter assisted wireless powered communications," IEEE Wireless Communications, vol. 25, no. 2, pp. 170-177, 2018.

[9] D. T. Hoang, D. Niyato, P. Wang, D. I. Kim, and Z. Han, "Ambient backscatter: a new approach to improve network performance for RF-powered cognitive radio networks," IEEE Transactions on Communications, vol. 65, no. 9, pp. 36593674, 2017.

[10] D. T. Hoang, D. Niyato, P. Wang, and D. I. Kim, "Optimal time sharing in rf-powered backscatter cognitive radio networks," in Proceedings of the 2017 IEEE International Conference on Communications (ICC), pp. 1-6, Paris, France, May 2017.

[11] B. Lyu, H. Guo, Z. Yang, and G. Gui, "Throughput maximization for hybrid backscatter assisted cognitive wireless powered radio networks," IEEE Internet of Things Journal, vol. 5, no. 3, pp. 2015-2024, 2018.

[12] R. Kishore, S. Gurugopinath, P. C. Sofotasios, S. Muhaidat, and N. Al-Dhahir, "Opportunistic ambient backscatter communication in rf-powered cognitive radio networks," IEEE Transactions on Cognitive Communications and Networking, vol. 5, no. 2, pp. 413-426, 2019.

[13] L. Shi, R. Q. Hu, J. Gunther, Y. Ye, and H. Zhang, "Energy efficiency for RF-powered backscatter networks using HTT protocol," IEEE Transactions on Vehicular Technology, vol. 69, no. 11, pp. 13932-13936, 2020.

[14] Y. Chen, N. Zhao, and M.-S. Alouini, "Wireless energy harvesting using signals from multiple fading channels," IEEE Transactions on Communications, vol. 65, no. 11, pp. 50275039, 2017.

[15] L. Tassiulas and S. Sarkar, "Maxmin fair scheduling in wireless ad hoc networks," IEEE Journal on Selected Areas in Communications, vol. 23, no. 1, pp. 163-173, 2005.

[16] S. Boyd, Convex Optimization, Cambridge University Press, Cambridge, UK, 2004. 